\title{
THE CONJUGATE PROPERTY FOR DIOPHANTINE APPROXIMATION OF CONTINUED FRACTIONS
}

\author{
JINGCHENG TONG
}

(Communicated by Williams W. Adams)

\begin{abstract}
Let $\xi$ be an irrational number with simple continued fraction expansion $\xi=\left[a_{0} ; a_{1}, \ldots, a_{i}, \ldots\right]$, and $p_{i} / q_{i}$ be its $i$ th convergent. In this paper we first prove the duality of some inequalities, and then prove the following conjugate properties for symmetric and asymmetric Diophantine approximations.

(i) Among any three consecutive convergents $p_{i} / q_{i}(i=n-1, n, n+1)$,
\end{abstract} at least one satisfies

$$
\left|\xi-p_{i} / q_{i}\right|<1 /\left(\sqrt{a_{n+1}^{2}+4} q_{i}^{2}\right) .
$$

and at least one does not satisfy this inequality.

(ii) Let $\tau$ be a positive real number. Among any four consecutive convergents $p_{i} / q_{i} \quad(i=n-1, n, n+1, n+2)$, at least one satisfies

$$
-1 /\left(\sqrt{c_{n}^{2}+4 \tau} q_{i}^{2}\right)<\xi-p_{i} / q_{i}<\tau /\left(\sqrt{c_{n}^{2}+4 \tau} q_{i}^{2}\right) .
$$

and at least one does not satisfy this inequality, where $c_{n}=a_{n+1}$ if $n$ is odd, $c_{n}=a_{n+2}$ if $n$ is even.

\section{INTRODUCTION}

Let $\xi$ be an irrational number with simple continued fraction expansion $\xi=\left[a_{0} ; a_{1}, \ldots, a_{i}, \ldots\right]$, and $p_{i} / q_{i}$ be its $i$ th convergent. A basic theorem on symmetric Diophantine approximation (see $[1,2,4])$ asserts that among any three consecutive convergents $p_{i} / q_{i} \quad(i=n-1, n, n+1)$, at least one satisfies

$$
\left|\xi-\frac{p_{i}}{q_{i}}\right|<\frac{1}{\sqrt{a_{n+1}^{2}+4 q_{i}^{2}}} .
$$

In 1983, the present author proved the conjugate property of the above theorem in [9]: among any three consecutive convergents $p_{i} / q_{i}$, at least one satisfies

$$
\left|\xi-\frac{p_{i}}{q_{i}}\right|>\frac{1}{\sqrt{a_{n+1}^{2}+4 q_{i}^{2}}},
$$

this fact was rediscovered by Prasad and Lari [6] in 1986.

Received by the editors December 18, 1987 and, in revised form, April 20, 1988.

1980 Mathematics Subject Classification (1985 Revision). Primary 11J04, 11 A55. 
Let $\tau$ be a positive real number. The present author [11] improved the results on asymmetric Diophantine approximation in $[3,7,8]$, and obtained a basic theorem: among any four consecutive convergents $p_{i} / q_{i}(i=n-1, n$, $n+1, n+2)$, at least one satisfies

$$
\frac{-1}{\sqrt{c_{n}^{2}+4 \tau} q_{i}^{2}}<\xi-\frac{p_{i}}{q_{i}}<\frac{\tau}{\sqrt{c_{n}^{2}+4 \tau} q_{i}^{2}},
$$

where $c_{n}=a_{n+1}$ if $n$ is odd, and $c_{n}=a_{n+2}$ if $n$ is even.

In this paper, we prove the duality of some inequalities, which gives a unified treatment on conjugate properties of both symmetric and asymmetric approximations. The method in [12] is used to prove that among any four consecutive convergents $p_{i} / q_{i}$, at least one satisfies one of the following inequalities:

$$
\begin{gathered}
\xi-\frac{p_{i}}{q_{i}}<\frac{-1}{\sqrt{c_{n}^{2}+4 \tau} q_{i}^{2}}, \\
\xi-\frac{p_{i}}{q_{i}}>\frac{\tau}{\sqrt{c_{n}^{2}+4 \tau} q_{i}^{2}} .
\end{gathered}
$$

\section{Preliminaries}

Let $M_{i}=\left[a_{i+1} ; a_{i+2}, \ldots\right]+\left[0 ; a_{i}, a_{i-1}, \ldots, a_{1}\right]$. It is well known that

$$
\xi-\frac{p_{i}}{q_{i}}=\frac{(-1)^{i}}{M_{i} q_{i}^{2}}
$$

Let $P=\left[a_{n+2} ; a_{n+3}, \ldots\right]$ and $Q=\left[a_{n} ; a_{n-1}, \ldots, a_{1}\right]$. It is easily seen that $P$ is an irrational number, $Q$ is a rational number. The following equalities are easy to check:

$$
\begin{gathered}
M_{n+1}=P+\frac{1}{a_{n+1}+Q^{-1}}, \\
M_{n}=a_{n+1}+\frac{1}{P}+\frac{1}{Q}, \\
M_{n-1}=Q+\frac{1}{a_{n+1}+P^{-1}} .
\end{gathered}
$$

We need an important lemma.

LEMMA 1 Let $a, r$ be two constants such that $0<a<r$, and $f(x)=$ $x+\frac{1}{r-x^{-1}}$. Then

(i) $f(x)>4 r /\left(r^{2}-a^{2}\right)$ for $x>2 /(r-a)$,

(ii) $f(x)<4 r /\left(r^{2}-a^{2}\right)$ for $2 /(r+a)<x<2 /(r-a)$.

Proof. Simple calculus. 


\section{DuALITY OF SOME INEQUALITIES}

Theorem 1. Let $r>a_{n+1}$. Then

(i) $M_{n}>r$ implies $\min \left(M_{n-1}, M_{n+1}\right)<4 r /\left(r^{2}-a_{n+1}^{2}\right)$.

(ii) $M_{n}=r$ implies $\min \left(M_{n-1}, M_{n+1}\right)<4 r /\left(r^{2}-a_{n+1}^{2}\right)$, and $\max \left(M_{n-1}, M_{n+1}\right)>4 r /\left(r^{2}-a_{n+1}^{2}\right)$.

(iii) $M_{n}<r$ implies $\max \left(M_{n-1}, M_{n+1}\right)>4 r /\left(r^{2}-a_{n+1}^{2}\right)$.

Proof. (i) If $M_{n}>r$, by (8) we have $P^{-1}>r-a_{n+1}-Q^{-1}$ and $Q^{-1}>$ $r-a_{n+1}-P^{-1}$. From (7) and (9), we have

$$
\begin{aligned}
& M_{n+1}<\frac{1}{r-P^{-1}}+P, \\
& M_{n-1}<\frac{1}{r-Q^{-1}}+Q .
\end{aligned}
$$

There are two possibilities on $P, Q$.

(a) One of $P, Q<2 /\left(r-a_{n+1}\right)$. Since $r>a_{n+1} \geq 1$, it is easily seen that $P>1>2 /\left(r+a_{n+1}\right)$ and $Q>2 /\left(r+a_{n+1}\right)$. By Lemma 1(ii), we have $\min \left(M_{n-1}, M_{n+1}\right)<4 r /\left(r^{2}-a_{n+1}^{2}\right)$.

(b) Both $P$ and $Q \geq 2 /\left(r-a_{n+1}\right)$. Since $P$ is irrational and $Q$ is rational, $P \neq Q$. Hence at least one of $P, Q>2 /\left(r-a_{n+1}\right)$, by (8) we have $M_{n}+a_{n+1}+$ $P^{-1}+Q^{-1}<r$, contradicting the assumption $M_{n}>r$.

Combining (a), (b), we have assertion (i).

(ii) If $M_{n}=r$, by (8) and (7), (9) we have

$$
\begin{aligned}
& M_{n+1}=\frac{1}{r-P^{-1}}+P, \\
& M_{n-1}=\frac{1}{r-Q^{-1}}+Q .
\end{aligned}
$$

By the above proof, we have $\min \left(M_{n-1}, M_{n+1}\right)<4 r /\left(r^{2}-a_{n+1}^{2}\right)$ by discussing the two cases (a) and (b).

We may also consider the following two cases on $P, Q$.

(a') One of $P, Q>2 /\left(r-a_{n+1}\right)$. By (12), (13) and Lemma 1(i), we have $\max \left(M_{n-1}, M_{n+1}\right)>4 r /\left(r^{2}-a_{n+1}^{2}\right)$.

(b') Both $P, Q \leq 2 /\left(r-a_{n+1}\right)$. Since $P \neq Q$, at least one of $P, Q<$ $2 /\left(r-a_{n+1}\right)$. By $(8)$ we have $M_{n}>r$, contradicting the assumption $M_{n}=r$.

Combining ( $\left.\mathbf{a}^{\prime}\right)$ and $\left(\mathbf{b}^{\prime}\right)$, we have assertion (ii).

(iii) If $M_{n}<r$, by (8) and (7), (9), we have

$$
M_{n+1}>\frac{1}{r-P^{-1}}+P \text {. }
$$




$$
M_{n-1}>\frac{1}{r-Q^{-1}}+Q
$$

Similar to the proof of assertion (ii), we have assertion (iii) by discussing the two cases $\left(a^{\prime}\right)$ and $\left(b^{\prime}\right)$.

Remark 1. The case (iii) in Theorem 1 has been discussed in [10] in a different way.

\section{Applications}

Letting $r=\sqrt{a_{n+1}^{2}+4}$, we have $4 r /\left(r^{2}-a_{n+1}^{2}\right)=\sqrt{a_{n+1}^{4}+4}$. By (6) and Theorem 1, we have the following theorem.

Theorem 2. Among any three consecutive convergents $p_{i} / q_{i}$ of $\xi \quad(i=n-$ $1, n, n+1)$, at least one satisfies inequality (1), and at least one satisfies inequality (2).

Let

$$
c_{n}= \begin{cases}a_{n+1} & \text { if } n \text { is odd } \\ a_{n+2} & \text { if } n \text { is even. }\end{cases}
$$

Let $\tau$ be a positive real number. If $r=\sqrt{c_{n}^{2}+4 \tau}$, then $4 r /\left(r^{2}-c_{n}^{2}\right)=$ $\sqrt{c_{n}^{2}+4 \tau} / \tau$. By (6) and Theorem 1 , we have the following theorem by discussing the two cases: $n$ is odd or $n$ is even.

Theorem 3. Among any four consecutive convergents $p_{i} / q_{i}$ of $\xi \quad(i=n-$ $1, n, n+1, n+2)$, at least one satisfies inequality (3), and at least one satisfies one of the inequalities (4) or (5).

\section{ACKNOWLEDGMENT}

The author thanks the referee sincerely for his valuable suggestions to improve this paper.

\section{REFERENCES}

1. F. Bagemihl and J. R. McLaughlin, Generalization of some classical theorems concerning triples of consecutive convergents to simple continued fractions, J. Reine Angew. Math. 221 (1966), 146-149.

2. É. Borel, Contribution à l'analyse arithmétique du continu, J. Math. Pures Appl. (9) (1903), 329-375.

3. W. J. LeVeque, On asymmetric approximations, Michigan Math. J. 2 (1953), 1-6.

4. M. Müller, Über die Approximation reeler Zahlen durch die Näherungsbruche ihres regelmässigen Kettenbruches, Arch. Math. 6 (1955), 253-258.

5. C. D. Olds, Note on an asymmetric Diophantine approximation, Bull. Amer. Math. Soc. 52 (1946), 261-263.

6. K. C. Prasad and M. Lari, A note on a theorem of Perron, Proc. Amer. Math. Soc. 97 (1986), 19-20. 
7. B. Segre, Lattice points in infinite domains and asymmetric Diophantine approximation, Duke Math. J. 12 (1945), 337-365.

8. P. Szüsz, On a theorem of Segre, Acta Arith. 23 (1973), 371-377.

9. J. Tong, The conjugate property of the Borel theorem on Diophantine approximation, Math. $\mathrm{Z}$. 184 (1983), 151-153.

10. A theorem on approximation of irrational numbers by simple continued fractions, Proc. Edinburgh Math. Soc. 31 (1988), 197-204.

11. _ Segre's theorem on asymmetric Diophantine approximation, J. Number Theory 28 (1988), 116-118.

12. Symmetric and asymmetric Diophantine approximations of continued fractions, Bull. Soc. Math. France. (to appear).

Department of Mathematical Sciences, University of North Florida, Jacksonville, Florida 32216 and Institute of Applied Mathematics, Academia Sinica, Beijing, China 